Relations industrielles

Industrial Relations

\title{
L'obligation de civilité du salarié
}

\section{Claude D'Aoust, Sylvain Saint-Jean et Gilles Trudeau}

Volume 41, numéro 1, 1986

URI : https://id.erudit.org/iderudit/050187ar

DOI : https://doi.org/10.7202/050187ar

Aller au sommaire du numéro

Éditeur(s)

Département des relations industrielles de l'Université Laval

ISSN

0034-379X (imprimé)

1703-8138 (numérique)

Découvrir la revue

Citer cet article

D'Aoust, C., Saint-Jean, S. \& Trudeau, G. (1986). L'obligation de civilité du salarié. Relations industrielles / Industrial Relations, 41(1), 157-180. https://doi.org/10.7202/050187ar
Résumé de l'article

Les auteurs cherchent à mettre en évidence les relations interpersonnelles qui naissent à l'occasion de l'exécution du travail et à montrer à quelles normes les parties au contrat de travail doivent se conformer dans ces relations
Tous droits réservés (C) Département des relations industrielles de l'Universite Laval, 1986
Ce document est protégé par la loi sur le droit d'auteur. L’utilisation des services d'Érudit (y compris la reproduction) est assujettie à sa politique d'utilisation que vous pouvez consulter en ligne.

https://apropos.erudit.org/fr/usagers/politique-dutilisation/ 


\title{
DROIT DU TRAVAIL
}

\section{L'obligation de civilité du salarié}

\author{
Claude D'Aoust \\ Sylvain St-Jean \\ Gilles Trudeau
}

Les auteurs cherchent à mettre en évidence les relations interpersonnelles qui naissent à l'occasion de l'exécution du travail et à montrer à quelles normes les parties au contrat de travail doivent se conformer dans ces relations

Le travail salarié suppose, la plupart du.temps, l'insertion dans un milieu de travail et, même si la division du travail n'y est pas pratiquée, des relations sociales entre les personnes travaillant dans un même lieu ou à une entreprise commune.

Certaines de ces relations sont encadrées juridiquement par le contrat individuel de travail ${ }^{1}$. Ce sont celles qui retiennent présentement notre attention.

Il nous semble utile de distinguer d'abord les relations que le salarié entretient avec les représentants de l'autorité, et d'autre part les rapports qu'il a avec ses compagnons de travail ${ }^{2}$. Dans le premier cas, l'un des agents est en autorité et les rapports seront nécessairement (mais non exclusivement) déterminés par la subordination juridique dans laquelle se trouve le préposé; à cela, il faut greffer une obligation mutuelle de respect. En second lieu, les relations du salarié avec ses pairs peuvent influer sur le comportement du groupe et par là se rattachent à son obligation de fournir un rendement satisfaisant ${ }^{3}$.

L'obligation d'obéissance qui découle de la subordination juridique et l'obligation de fournir la prestation convenue, dont il a été fait état, sont des obligations essentielles à tout contrat de travail ${ }^{4}$. Mais cette liste n'est pas exhaustive et l'article 1024 du Code civil l'indique clairement: certaines obligations découlent de la nature même du contrat. Notre propos est de montrer que la civilité est une obligation naturelle du salarié, particulièrement lorsque l'exécution du travail s'accomplit dans un milieu de travail constituant un groupe social. Il n'est pas question ici d'ajouter une nouvelle obligation à la charge du salarié, mais simplement de mettre en évidence certains aspects de ses obligations qui entrent en ligne de compte dans des

* D'AOUST, C., S. ST-JEAN et G. TRUDEAU, professeurs, Ecole de relations industrielles, Université de Montréal

** Toutes les décisions mentionnées ci-après ont été analysées dans le texte intégral.

*** Les auteurs remercient madame la professeure Christiane Louis-Guérin, pour ses précieux commentaires concernant la première partie de cet article.

Relat. ind., vol. 41, no 1, 1986 ㄷ PUL ISSN 0034-379 X 
situations particulières. D'ailleurs, il en sera de même pour certains devoirs incombant à l'employeur, lequel, à l'occasion, pourra être appelé à prendre parti pour l'individu ostracisé pour des motifs prohibés par la loi.

À ce stade, il importe cependant d'éclairer le lecteur sur le choix de notre vocabulaire. Nous nous proposons de dégager les devoirs du salarié en tant que membre d'un groupe social. Or, la civilité est définie par le Grand dictionnaire encyclopédique Larousse ( 1 er sens):

«Observation des convenances en usage chez les gens qui vivent en société; politesse, courtoisie (vieilli)...»

Le Petit Robert lui donne lui aussi comme premier sens (vieilli): "Observation des convenances, des bonnes manières en usage dans un groupe social.»

Enfin, le Larousse de la langue française (Lexis) définit la civilité de la même façon.

Nous retiendrons donc ce terme ${ }^{5}$. Le lecteur nous permettra cependant deux remarques.

D'abord si le terme est vieilli, il n'en va pas nécessairement de même de son contenu. Les règles de comportement changent avec les milieux et les époques, mais ces règles sont toujours présentes en milieu social.

Deuxièmement, il faut noter l'emploi du mot «usage» dans les deux définitions rapportées. Il ne s'agit pas ici de juger le comportement du salarié à partir de critères étrangers à son milieu de travail mais bien en fonction de règles issues du milieu lui-même.

D'autre part, nous avons rejeté l'expression «devoir de coopération» vu que depuis longtemps déjà le droit lui a donné un sens précis et général s'appliquant à l'esprit dans lequel doit s'exécuter tout contrat, quelle qu'en soit la nature ${ }^{6}$.

Par ailleurs, l'obligation de civilité transcende la stricte notion de productivité. Elle touche également les rapports simplement humains. Elle suppose que c'est à l'individu de s'adapter au groupe social et, en cas de refus, l'employeur peut être appelé à déplacer ou renvoyer l'individu avant qu'il ne soit rejeté par le groupe?

Quant à l'obligation d'obéissance, au-delà du simple respect de l'autorité hiérarchique, elle suppose complémentairement un devoir de respect et de loyauté à l'égard des personnes investies de l'autorité ${ }^{8}$, sans lesquels l'obédience manifestée ne saurait qu'être éphémère et factice. Par ailleurs, ce respect ne doit pas être une façade et le salarié ne doit rien faire qui soit de nature à miner l'autorité de son supérieur dans l'esprit de ses compagnons de travail.

Notre étude a pour but de mettre en évidence les relations interpersonnelles qui naissent à l'occasion de l'exécution du travail et de montrer à quelles normes les parties au contrat de travail doivent se conformer dans ces relations. C'est donc du comportement du salarié à l'intérieur du groupe qu'il s'agit et des sanctions qu'entraîne la violation de ces normes. La sanc- 
tion peut provenir du groupe social lui-même; nous pensons ici au rejet de l'individu par ses compagnons de travail. Nous en parlerons dans une première partie. Mais, ces perturbations peuvent nuire au rendement individuel ou collectif entrainant des sanctions de la part de l'employeur, cellesci étant régies par le droit du travail cette fois; dans la deuxième partie nous examinerons certaines décisions judiciaires ou sentences arbitrales relatives à ce type de problème.

\section{L'INSERTION DE L'INDIVIDU DANS UN GROUPE SOCIAL ET SES CONSÉQUENCES}

Afin de cerner les fondements de l'obligation de civilité, nous ferons un rappel des notions développées par la discipline qui s'intéresse aux relations de l'individu avec le groupe auquel il appartient, soit la psychologie sociale ${ }^{9}$.

Dans la suite du texte, nous définirons par rapport au milieu de travail ce qu'est un groupe, comment il se forme, et en quoi l'une des principales caractéristiques des groupes, à savoir l'existence de normes, se rattache à l'obligation de civilité. Â cette étape, nous décrirons le processus par lequel le salarié adhère aux normes du groupe, et à quoi il s'expose s'il refuse ou fait défaut de s'y conformer.

\section{Définition du groupe}

La première étape consiste à savoir comment la psychologie sociale définit le groupe et si cette définition est applicable à un groupe de salariés.

Malgré quelques divergences sur des aspects mineurs de la définition du groupe, celle qu'ont retenu les auteurs ${ }^{10} \mathrm{~s}^{\prime}$ applique aux groupes existant en milieu de travail. Le groupe est constitué d'un certain nombre d'individus, ayant des relations entre eux, conscients de former un groupe distinct, et travaillant dans un but commun.

\section{Formation du groupe}

Déjà à cette étape de la discussion, nous devons distinguer deux types de groupes au sein de l'entreprise: le groupe formel et le groupe informel ${ }^{11}$. Bien que les individus qui composent le premier groupe puissent aussi faire partie du second, la distinction entre ces deux groupes est tout de même primordiale aux fins de l'analyse. Nous comparerons les deux types, bien que l'obligation de civilité s'applique principalement à l'observation par le salarié des normes du groupe informel, vu que les liens y sont plus serrés et les relations plus suivies.

\section{Constitution du groupe formel}

En milieu de travail, les individus sont réunis au sein de l'entreprise dans le but d'en réaliser les objectifs: produire un bien ou offrir un service. Chaque individu accepte, par le truchement du contrat individuel de travail, 
de fournir la prestation de travail convenue, et dans le cas qui nous occupe, de s'associer à d'autres individus, conformément à l'ordonnance des tâches à accomplir. Les raisons qui motivent une personne à accepter tel ou tel emploi ne sont pas, ici, pertinentes.

\section{Constitution du groupe informel}

Parmi l'ensemble des travailleurs d'une entreprise et parallèlement aux groupes formés par l'employeur selon les exigences de la production, se créent des groupes informels en raison du besoin social de chaque individu. Comme le constate un auteur:

«Because the whole person actually reports for work or joins the organization, and because people have needs beyond the minimum ones of doing their job, they will seek fulfillment of some of these needs through developing a variety of relationships with other members of the organization. If the ecology of the work area and the time schedule of the work permit, these informal relationship will develop into informal groups. In other words, the tendency toward informal groups can almost always be assumed to exist because we all have needs to relate to other people.»12

La proximité, les interactions et les communications entre les individus, les caractéristiques communes, le besoin d'affiliation, l'expression et la défense de revendications communes, etc., sont autant de facteurs expliquant la formation des groupes informels ${ }^{13}$.

Précisons que lorsqu'une entreprise est créée et que plusieurs salariés sont embauchés au même moment, la constitution des groupes informels se fait par étapes, mais puisque l'obligation de civilité concerne les relations de l'individu avec le groupe, nous n'insisterons pas sur cet aspect de la question $^{14}$. Nous postulerons plutôt que le groupe auquel doit s'intégrer le salarié possède déjà les caractéristiques d'un groupe pleinement développé. Le lecteur gardera toutefois en mémoire que le groupe et les caractéristiques qui s'y rattachent sont des notions dynamiques qui, au fil du temps, ne cessent d'évoluer et de se transformer.

\section{Les normes du groupe}

Qu'ils soient formels ou non, les groupes possèdent diverses caractéristiques ${ }^{15}$ : une taille, un statut et un rôle défini pour chaque membre, un réseau particulier de communication, un leadership exercé par un ou plusieurs membres, un certain degré de cohésion et d'efficacité face aux tâches à accomplir, des objectifs, un but, etc. Parmi ces caractéristiques, l'existence de normes au sein des groupes doit être analysée pour préciser le sens de l'obligation de civilité.

Plusieurs auteurs proposent une définition des normes ${ }^{16}$ et celle de Hackman semble faire l'unanimité:

«There is general agreement that a norm is a structural characteristic of a group which summarizes and highlights those processes within the group which are intended to regulate and regularize group member behavior. . ${ }^{17}$ 
En d'autres termes, les normes établissent le comportement auquel s'attend le groupe de la part de chacun de ses membres dans des circonstances particulières. Ces comportements dictés par les normes peuvent concerner le geste ou la parole.

Dans une société donnée, envisagée comme le groupe social le plus large, certaines normes formelles existent: c'est le droit objectif. D'autres, bien que demeurant informelles, reposent sur les valeurs communes de la société et l'individu qui y déroge s'expose à la réprobation de son entourage.

Outre ces normes générales, on retrouve à l'échelle réduite des normes spécifiques dans chaque groupe de travail. De plus, il faut préciser, qu'il y $\mathrm{a}$, du point de vue du groupe des normes plus importantes que d'autres, de telle sorte que la réprobation qui s'exerce envers le membre, varie en fonction de la norme à laquelle il a dérogé ${ }^{18}$. À ce titre d'ailleurs, c'est également une caractéristique des normes que celles-ci ne s'appliquent pas uniformément à tous les membres du groupe, certains d'entre eux, de par leur statut (les leaders par exemple) jouissant de certains privilèges.

L'application de ces notions aux groupes formels et informels de travail, ainsi que quelques exemples permettront de le mieux comprendre.

\section{Les normes du groupe formel}

Dans ce groupe, les normes les plus importantes sont fixées par l'employeur de façon formelle à travers les clauses du contrat de travail ou de la convention collective et par les politiques et règlements d'entreprise ${ }^{19}$. Ainsi sont établis pour chaque membre du groupe la tâche à accomplir, la façon de l'accomplir, le rendement qu'il doit fournir, sa position dans l'organisation du travail et ses rapports formels avec les divers paliers de l'autorité, ainsi qu'avec les autres travailleurs, son horaire de travail, le moment et la durée des pauses, repas, congés et vacances, etc.

Au moment de l'embauchage, le salarié accepte de respecter les normes, et en vertu du lien de subordination juridique, toute dérogation de sa part autorise l'employeur à imposer des sanctions. Quant aux normes d'importance secondaire, elles sont également établies par l'employeur, mais cette fois de façon informelle. Elles concernent les comportements évalués par l'employeur, sans qu'une dérogation n'entraîne nécessairement l'imposition d'une sanction. Ce sont l'ardeur et la minutie au travail, la promptitude à exécuter les ordres, l'identification aux objectifs de l'entreprise, le dynamisme, l'initiative, etc. (sauf s'il existe un système complexe d'évaluation au mérite).

L'observation de ces normes permet, aux yeux de l'employeur, de distinguer le salarié exemplaire du salarié moyen.

\section{Les normes du groupe informel}

Malgré l'apparente rigidité des normes fixées par l'employeur, le salarié, selon le poste qu'il occupe et la complexité des tâches qui lui incombent, jouit de plus ou moins d'autonomie dans son travail et en regard des 
relations qu'il peut établir avec les autres. Tout comme la constitution du groupe informel se fait parallèlement aux groupes créés par l'employeur en conformité avec les exigences de la production, les normes du groupe informel se développent en marge de celles fixées par l'employeur, et peuvent même, dans certains cas, aller à l'encontre de celles-ci ${ }^{20}$. Les normes du groupe informel concernent non seulement une foule d'aspects de la vie au travail et des relations des salariés entre eux ainsi qu'avec les représentants de l'autorité, mais également les comportements du salarié à l'extérieur du travail.

Puisque les normes du groupe informel ne sont pas écrites, il est toujours difficile pour l'observateur extérieur de les identifier, d'autant plus qu'elles sont pratiquement illimitées et qu'elles varient d'un groupe à l'autre en fonction des milieux de travail. Les normes du groupe informel concernent, par exemple, les quotas de production, l'exécution du travail (aider ou non les autres, laisser son poste de travail en bon état et sans travail en retard pour celui qui entreprend le prochain quart, prêter ou non ses outils), l'attitude envers les supérieurs hiérarchiques, les absences-maladie (éviter de s'absenter quand le groupe doit faire l'inventaire, nettoyer l'usine, etc. ou lorsque des salariés sont en vacances), les activités sociales du groupe (participer aux rencontres sociales organisées par le groupe), le comportement des membres à l'extérieur du travail (ne pas faire l'objet de poursuites criminelles, ne pas discréditer la qualité du travail des membres), etc. En somme, le salarié doit respecter les us et coutumes du groupe qui englobent également des objets non directement reliés au travail, tels que la façon de se vêtir, le niveau de langage à respecter, l'intrusion dans la vie privée d'un compagnon de travail, etc. ${ }^{21}$.

Ici, la différenciation des normes selon leur ordre d'importance est difficile à faire, chaque cas étant d'espèce. On peut présumer, par exemple, que les normes relatives aux quotas de production, à l'exécution du travail et aux comportements envers les autres membres doivent être plus importantes, alors que celles relatives aux activités sociales du groupe et aux comportements des membres à l'extérieur du travail doivent l'être moins.

\section{Le processus d'acquisition des normes: la socialisation}

Dès le moment où l'individu entre en emploi ou change de travail (par promotion, mutation ou autrement) s'amorce un processus par lequel il acquiert les normes du groupe. Ce processus, appelé socialisation, est le même à l'échelle réduite du groupe, que celui qui agit sur l'individu dès son entrée dans la société, c'est-à-dire à la naissance:

"Socialization involves the process by which a person acquires, from the enormously wide range of behavioral potentialities that are open to him or her starting at with those behavior patterns that are customary and acceptable according to the standards of, initially, the family, and later the social group and the employing organization (...) the process includes only the learning of those values, norms, and behavior patterns which, from the organization's work group's point of view are necessary for any new organization member to learn". ${ }^{22}$

Schein décrit de façon plus précise la socialisation en milieu de travail: 
$\ll \ldots$ socialization refers to the process of teaching the new recruit how to get along in the organization, what the key norms and rules of conduct are, and how to behave with respect to others in the organization - what is often referred to as «learning the ropes». The new recruit must learn where to be at specified times, what to wear, what to call the boss, whom to consult if he or she has a question, how carefully to do a job, and endless other things which insiders have learned over time. ${ }^{23}$

La socialisation du salarié s'effectue simultanément sur deux plans. D'une part, elle conduit à l'acquisition des normes du groupe formel fixées par l'employeur, et d'autre part à l'acquisition de celles du groupe informel développées par ce dernier.

Le processus de socialisation doit conduire à l'établissement d'un «contrat psychologique» entre le salarié et les représentants de l'autorité, ainsi qu'avec les autres salariés:

"The notion of psychological contract implies that there is an unwritten set of expectations operating at all times between every member of an organization and the various managers and others in that organization. ${ }^{24}$

Les raisons qui peuvent faire en sorte qu'un tel contrat psychologique ne s'établisse pas entre le salarié et le groupe sont multiples. En regard de l'obligation de civilité, il y a problème si le contrat psychologique ne s'établit pas, parce que le salarié, par ses comportements et attitudes, refuse de se conformer aux normes du groupe. Dans le cas du groupe formel, la dérogation aux normes fixées par l'employeur entraîne la rétorsion de ce dernier au moyen de mesures patronales (disciplinaires ou administratives) dont l'application est bien connue. Par contre, lorsqu'il y a dérogation aux normes du groupe informel, le réprobation s'exerce par le groupe de diverses façons, comme le harcèlement verbal et physique, la noncoopération, le sabotage de son matériel, la diminution ou l'arrêt des communications et des échanges interpersonnels, la dévalorisation de son travail au sein du groupe, etc.

Les effets de sa réprobation par le groupe du comportement du salarié, ou en d'autres termes les effets de l'absence de contrat psychologique entre le salarié et le groupe, se font sentir sur deux plans. D'abord chez le salarié, dont les réactions à ce rejet de la part du groupe peuvent être multiples: démission, rêverie, apathie, perte d'intérêt au travail, stress, insatisfaction, alcoolisme, agressivité envers les autres, assaut, absentéisme, etc. Ensuite, au sein du groupe alors que la cohésion, l'harmonie, le leadership, la communication, en somme toutes ces caractéristiques essentielles a l'efficacité et à la qualité du travail de groupe sont perturbées.

\section{LE DROIT POSITIF ET L'OBLIGATION DE CIVILITÉ DU SALARIÉ}

Dans cette seconde partie, nous scruterons les sources du droit positif dans le but de vérifier si une telle obligation de civilité a été reconnue dans le cadre du contrat individuel de travail. Nous discuterons, le cas échéant, de son étendue et des circonstances dans lesquelles elle se manifeste. Enfin, nous analyserons la sanction juridique qui peut découler du non-respect 
d'une telle obligation. En d'autres termes, comment un employeur, créancier de l'obligation de civilité à la charge du salarié, peut-il réagir à l'endroit du débiteur en défaut?

À cet effet, la jurisprudence constitue certes la source de droit privilégiée. Le législateur, pour sa part, n'a pas imposé explicitement cette obligation; c'est plutôt par l'intermédiaire de l'article 1024 du Code civil qu'elle découlerait de la nature du contrat de travail. Cet article se lit:

«1024. Les obligations d'un contrat s'étendent non seulement à ce qui est exprimé, mais encore à toutes les conséquences qui en découlent d'après sa nature et suivant l'équité, l'usage ou la loi.»"

Il demeure cependant que le législateur a, à l'occasion, touché à des aspects particuliers de l'obligation de civilité. Il l'a fait surtout en prohibant certaines formes de harcèlement et de discrimination en milieu de travail par des législations plus larges visant les droits de la personne. Ainsi, le rejet du salarié par le groupe social ne pourra être interprété comme un manquement à l'obligation de civilité si ce rejet est le fruit de discrimination ou de harcèlement prohibés par ces lois. Nous y reviendrons. Nous avons aussi puisé dans la doctrine, rare sur le sujet, pour soutenir notre raisonnement.

Quant à la jurisprudence étudiée, elle vient de plusieurs sources. Les décisions arbitrales constituent une source privilégiée à cause de leur nombre, leur richesse et la diversité des situations qu'elles embrassent. Nous avons regardé aussi bien du côté de l'arbitrage des griefs que du côté des décisions rendues en vertu de l'article 61.5 de la Partie III du Code canadien du travail ${ }^{25}$ ou des articles 124 et seq. de la Loi sur les normes du travail du Québec ${ }^{26}$. Nous avons de plus fait de brèves incursions dans la jurisprudence provenant des tribunaux québécois de droit commun et aussi des tribunaux des autres provinces canadiennes ${ }^{27}$. Nous n'avons cependant aucunement la prétention d'être exhaustifs. Cette réserve doit d'ailleurs être ajoutée à une autre qui relève directement de l'objet de notre étude: 1'incertitude et la variété des opinions émises à l'endroit de situations toutes plus particulières les unes que les autres et qui relèvent toujours en premier lieu de l'attitude des personnes impliquées. Ces deux réserves atténuent la portée de la généralisation que nous présentons dans les prochaines pages.

\section{Existence de l'obligation de civilité et manquements qui lui sont associés}

Il semble bien que l'obligation de civilité soit reconnue dans notre droit. Un salarié doit coopérer avec ses collègues de travail, il doit être poli envers eux, bref il doit s'efforcer de maintenir des relations harmonieuses avec eux. L'impossibilité de remplir ce devoir pourra justifier l'employeur de mettre fin à l'emploi du salarié28. C'est ainsi que dans une décision récente de l'Ontario Supreme Court, on retrouve le passage suivant:

«... an employer is entitled to dismiss an employee who has been warned from time to time that he has an incompatible personality conflict but refuses or is unable to improve or correct his relationships and similar incidents later.» ${ }^{29}$ 
Une reconnaissance aussi formelle de l'obligation de civilité mérite d'être nuancée. Auparavant, voyons comment le défaut de respecter cette obligation peut apparaître. Généralement, c'est le comportement du salarié qui suscitera la réaction négative des personnes avec lesquelles il travaille. Cette réaction s'exprimera par des plaintes auprès de l'employeur, des pétitions ou menaces à l'effet que dorénavant on ne veut plus travailler en leur compagnie, etc. On peut même penser à des griefs déposés auprès de l'employeur en vertu d'une convention collective pour contester les gestes d'une personne par ailleurs en autorité à l'endroit d'un ou de plusieurs salariés syndiqués ${ }^{30}$.

Nous retrouvons dans la jurisprudence plusieurs exemples de comportements qui ont suscité une réaction négative du groupe social auquel appartenait le salarié contesté. La vulgarité, la grossièreté, les excès de langage et la rudesse à l'endroit des compagnons de travail sont souvent à la base des fautes d'incivilité ${ }^{31}$. Adopter une attitude négative constante à l'endroit du travail des autres, les traiter d'imbéciles, se prétendre le seul salarié à effectuer correctement le travail sont d'autres comportements pouvant engendrer des problèmes de rejet ${ }^{32}$.

Le refus de coopérer et de travailler en équipe s'avère aussi une source de dissension au sein du groupe social. Par exemple, dans Re Continuous Colour Coat Ltd. c. United Steel Workers, on reprochait au salarié des pauses-café beaucoup trop longues, des heures de lunch prises même au moment où sa présence était requise sur la ligne de montage et aussi un manque de coopération avec les autres travailleurs dans certaines tâches, comme le nettoyage, alors que la coopération à cet égard était la pratique établie. En maintenant le congédiement, l'arbitre écrit:

"In this case, the grievor's proven behaviour did manifest an attitude of selfish concern with his own interests at the expense of those of his fellow workers and his employer, an attitude of stubborn insistence on his rights as he conceived of them, again to the detriment of his fellow workers and employer.

\section{(...)}

I find that the employer, in the result, has not erred in determining that the grievor's attitude disabled him, or was likely to disable him, from properly performing his job). ${ }^{33}$

Ailleurs, un arbitre a confirmé le congédiement d'une salariée qui avait rendu le climat complètement intolérable dans les cuisines du centre hospitalier où elle travaillait. Ses agissements imprévisibles et brusques, qui avaient même à l'occasion mis en danger certaines de ses compagnes de travail, son manque de communication et de collaboration, sa méfiance, son agressivité et ses traits de personnalité schizoïdes témoignaient de son incapacité à travailler en équipe. Le climat de tension était tel que les employés avaient menacé de débrayer pour forcer l'employeur à réagir à son comportement. ${ }^{34}$

Une personne peut aussi perturber le fonctionnement du groupe en y semant la discorde. Ainsi, contreviendrait à l'obligation de civilité le travailleur qui serait l'instigateur de différents conflits interpersonnels dans le groupe ${ }^{35}$. Faire signer par les clients une pétition contre un autre salarié de l'entreprise s'apparenterait au même comportement ${ }^{36}$. 
Le conflit de personnalités entre un salarié et son supérieur immédiat soulève un autre aspect de la civilité au travail. Il ne s'agit pas ici du salarié qui est rejeté par son groupe social au travail mais plutôt de deux individus ayant à travailler ensemble, dans une situation d'autorité l'un par rapport à l'autre, et qui ne parviennent pas à s'entendre. De plus, le conflit n'est pas causé par le refus du subalterne de reconnaître l'autorité de son supérieur, ce qui équivaudrait alors à de l'insubordination. Il semble bien que la jurisprudence refuse dans un tel cas de faire supporter au seul salarié les conséquences néfastes de ce conflit interpersonnel. En d'autres termes, ce dernier n'est pas débiteur d'une obligation de résultat de bien s'entendre avec son supérieur. Le conflit de personnalités avec le supérieur hiérarchique ne constitue donc pas en soi une cause valable de congédiement ${ }^{37}$.

D'autre part, il en va autrement si le conflit de personnalités est causé par la mauvaise volonté du salarié à l'endroit de son supérieur. Par exemple, un cadre supérieur qui déclare que dorénavant il refusera de travailler avec le secrétaire général de l'organisation commet une faute susceptible de provoquer son congédiement ${ }^{38}$. De la même façon, une attitude intempestive et agressive à l'endroit du supérieur immédiat constitue certainement une dérogation aux devoirs du salarié ${ }^{39}$.

Par ailleurs, si le supérieur immédiat exerce son autorité de façon inadéquate ou intrasigeante a l'endroit du salarié, la faute de ce dernier sera elle-même amoindrie si non complètement effacée ${ }^{40}$. Quant au supérieur hiérarchique, il pourra être blâmé si son comportement fautif ou l'exercice inconsidéré de son pouvoir de direction est à la base d'un conflit de personnalités avec un subalterne ou de son rejet par ses compagnons de travail ${ }^{41}$.

Nous mentionnions plus haut que, souvent, le rejet d'un salarié par le groupe auquel il appartient est communiqué à l'employeur par l'intermédiaire d'une pétition ou d'une requête du groupe à l'effet que, dorénavant, on refuse de travailler avec ou auprès de telle personne. Quelle est la valeur qui peut être accordée à ce document ou cette démarche? D'un point de vue procédural, une pétition écrite pourra être admissible en preuve devant les tribunaux sous certaines conditions. Devant les tribunaux de droit commun. un tel document devra être débosé par ses signataires. Autrement la pétition constituera une preuve par ouî-dire inadmissible. Ainsi en va-t-il des plaintes ou menaces de cesser de travailler formulées par des salariés membres du groupe. Pour les prouver, il faudra faire témoigner leurs auteurs.

La règle est quelque peu simplifiée devant les tribunaux d'arbitrage. On sait que l'arbitre est maître de la procédure et de la preuve à l'audition ${ }^{42}$. À ce titre, il peut décider de l'admissibilité de la pétition en soi et de sa valeur probante sans être tenu de respecter strictement les règles de preuve prévalant en droit commun. Ainsi dans l'affaire Charron c. Madras et al, la Cour d'appel du Québec a confirmé à l'instar de la Cour supérieure une décision arbitrale maintenant le congédiement d'un salarié qui avait fait l'objet d'une pétition de la part de ses compagnons de travail. La décision arbitrale s'appuyait, entre autres, sur cette pétition qui avait été produite par l'employeur sans faire témoigner les signataires et ce, malgré l'objection syndicale. Les tribunaux supérieurs ont confirmé qu'il appartenait à l'ar- 
bitre de décider de la recevabilité de la preuve à l'instruction du grief. De plus, aucun déni de justice ou accroc à la justice naturelle n'avait été établi par le syndicat ${ }^{43}$.

Une fois admise en preuve, la pétition des salariés ne saurait lier l'arbitre. De la même façon, l'employeur ne pourrait réagir aveuglément à partir du seul voeu exprimé par les autres salariés. Comme le mentionnait l'arbitre Deslierres, il n'appartient pas au personnel d'une entreprise de décider du sort d'un travailleur ${ }^{44}$. Cependant, une pétition peut certainement avoir un poids considérable, compte tenu des circonstances. Les propos suivants tirés d'une décision de l'arbitre Turcotte sont intéressants dans cette perspective:

«La pétition du 30 mai 1979, signée par une quinzaine d'employés, demandant le transfert de $\mathrm{M}$. Tourigny à un autre service que celui de l'entretien ménager, vu sa grossièreté envers les employés, est symptomatique, selon nous, du peu d'estime, du peu de sympathie que même les compagnons de travail de $\mathrm{M}$. Tourigny ont à l'égard de ce dernier.

Quand un salarié, par sa conduite, ses gestes, son attitude générale, s'attire la réprobation de deux-tiers de ses confrères de travail,à un point tel qu'on demande le renvoi du porteur du grief de l'équipe dans lequel il se trouve, il faut qu'il y ait définitivement anguille sous roche, et conduite réellement anormale d'un employé qui est rejeté même par ses compagnons de travail.

Un employé ne peut avoir raison envers et contre tous, et ce principalement tant à l'égard de son employeur qu'à l'égard de l'équipe d'entretien dont il fait partie».4.

\section{Étendue de l'obligation de civilité}

Nous avons montré l'existence d'une obligation de civilité découlant du contrat individuel de travail. Mais quelle en est la portée?

L'étendue de cette obligation varie beaucoup selon les circonstances. Le devoir à la charge du salarié sera d'autant plus grand que le travail à exécuter nécessite une étroite collaboration entre les membres du personnel $^{46}$. En fait, si la fonction du salarié exige des qualités de sociabilité qu'il ne possède pas, on pourra même parler d'incapacité ou d'inaptitude professionnelle $e^{47}$. Le milieu de travail comme tel doit aussi être pris en considération. Comme l'écrivait un arbitre, un hôpital est différent d'un chantier de construction par rapport à la civilité exigée des salariés entre eux ${ }^{48}$.

En second lieu, il n'y aura contravention à l'obligation de civilité que si le comportement du salarié est inadéquat ou fautif. Dans cette perspective, c'est davantage les attitudes et gestes du salarié lui-même que l'état de ses relations avec ses compagnons de travail qui devront être analysés pour découvrir s'il y a eu bris de l'obligation de civilité. Ainsi, un congédiement ne pourrait être justifié par le simple fait qu'un travailleur a cessé de plaire au groupe à l'intérieur duquel il travaille ${ }^{49}$. De la même façon, le seul conflit de personnalités entre deux personnes, même en état de subordination l'une par rapport à l'autre, ne saurait valablement fonder une brusque rupture du lien d'emploi ${ }^{50}$. Quant au comportement fautif qui pourrait être reproché au salarié et constituer un manquement à l'obligation de civilité, nous en avons donné des exemples plus haut ${ }^{31}$. 
Enfin, l'obligation de civilité du salarié n'est aucunement en cause lorsque ce dernier est rejeté par les autres membres du personnel pour des considérations prohibées par les différentes lois des droits de la personne ${ }^{52}$. Dans un tel cas, l'employeur poserait un geste discriminatoire en imposant une sanction ou même en prenant une simple mesure administrative (un transfert de département, par exemple) à l'endroit du salarié victime d'ostracisme. Il revient à l'employeur d'assurer au salarié un cadre de travail non discriminatoire ${ }^{53}$. C'est donc à l'endroit des autres salariés que l'employeur devra agir dans le but de les amener à modifier leurs attitudes à l'égard de la victime du rejet par le groupe.

Ce raisonnement s'applique aussi aux questions de harcèlement sexuel au travail. Les tensions causées entre une personne et un autre salarié (supérieur hiérarchique ou non) ou un groupe de salariés par du harcèlement sexuel ne peuvent être reprochées à la victime de cette pratique. Le harcèlement sexuel est expressément prohibé par nos lois sur les droits de la personne ${ }^{54}$. Cette pratique doit donc être traitée par l'employeur comme toute autre pratique discriminatoire illégale. À cet effet, le législateur fédéral a apporté des précisions supplémentaires en insérant dans la Partie III du Code canadien du travail les dispositions suivantes:

\subsection{Tout employé a droit a un emploi exempt de harcèlement sexuel}

61.9 L'employeur veille, dans toute la mesure du possible, à ce qu'aucun employé ne soit l'objet de harcèlement sexuel ${ }^{55}$.

Bien qu'aucun texte législatif équivalent n'existe dans la juridiction québécoise, nous croyons que les mêmes obligations sont implicitement imposées à l'employeur par la Charte des droits et libertés de la personne.

Toutes ces remarques nous permettent maintenant de conclure qu'il découle de la nature du contrat individuel de travail une obligation de civilité à la charge du salarié, selon l'article 1024 du Code civil. Cette obligation en est une de diligence et non de résultat. De plus, il semble que cette obligation survive à l'avènement d'une convention collective comme en font foi plusieurs décisions arbitrales ${ }^{56}$.

Les conséquences des manquements à l'obligation de civilité

La nature de l'obligation de civilité exige de l'employeur beaucoup de prudence lorsqu'il décide d'intervenir en ce domaine. N'oublions pas qu'il s'agit ici de répondre à des attitudes plutôt qu'à des gestes concrets et identifiés. Ceci nous amène d'ailleurs à faire une distinction entre les règles établies par les tribunaux de droit commun, et les règles élaborées par les arbitres de griefs et ceux qui agissent en vertu des articles 124 et seq de la $\mathbf{L o i}$ sur les normes du travail du Québec ou de l'article 61.5 de la Partie III du Code canadien du travail.

Généralement, un tribunal de droit commun est appelé à analyser les obligations découlant d'un contrat individuel de travail à la suite d'une action en dommages-intérêts intentée par un salarié congédié. En défense à cette action, l'employeur tentera de démontrer l'existence d'une cause de congédiement. Le tribunal se demandera s'il y a une inexécution d'une obliga- 
tion contractuelle, inexécution grave au point de justifier la résiliation unilatérale de contrat ${ }^{57}$. Ce cadre d'analyse laisse peu de latitude au tribunal dans l'appréciation de la proportionnalité entre la gravité de l'acte fautif et celle de la sanction imposée au salarié. Si le geste du salarié est suffisamment grave, le congédiement est fondé et l'action est rejetée. Au contraire, si la cause ne revêt pas ce nécessaire caractère de gravité, le congédiement est annulé et des dommages-intérêts sont accordés. En aucun cas, le tribunal ne peut substituer une mesure disciplinaire moins grave.

Il n'est guère surprenant que, vu la rigidité de ce cadre juridique, plusieurs décisions jurisprudentielles aient affirmé qu'un conflit de personnalités pouvait justifier un congédiement. Ainsi, dans Thormeloe c. La Commission scolaire régionale Eastern Townships ${ }^{58}$, la Cour supérieure décida que c'était à juste titre que l'employeur reprochait au salarié son attitude agressive à l'endroit de son supérieur mais que ces reproches ne pouvaient justifier un congédiement. La Cour ordonna donc à l'employeur de payer au salarié une indemnité représentant le salaire qu'aurait reçu le demandeur pendant la durée d'un préavis d'une longueur raisonnable. De la même façon, un employeur ne pourrait valablement congédier un salarié qui a de la difficulté à fonctionner à l'intérieur de son groupe de travail. La situation serait toutefois différente si ces difficultés causaient des problèmes sérieux à la bonne marche de l'entreprise ${ }^{59}$.

L'employeur faisant face à des problèmes de relations interpersonnelles parmi sa main-d'oeuvre ne pourra donc, en vertu des règles de droit commun, congédier sur-le-champ le salarié rejeté. Si la cessation d'emploi s'avère nécessaire, un préavis devra être donné au salarié (ou une indemnité en tenant lieu), en autant qu'il ait été engagé pour une durée indéterminée; par contre, s'il s'agit d'un contrat à terme, l'employeur devra garder le salarié à son service jusqu'à l'expiration de ce contrat. Il pourrait en être autrement si l'employeur démontrait que l'efficacité de son entreprise est sérieusement menacée ou encore que le rejet du salarié est sérieusement menacé ou encore que le rejet du salarié est causé par un manquement sérieux de sa part.

La situation pourrait quelque peu évoluer en droit commun dans la mesure où les tribunaux accepteront de réduire la durée du préavis lorsque la conduite du salarié, sans justifier un congédiement, n'est pas complètement sans reproche (notion de near cause developpée en common law). Ainsi, dans l'affaire Smith c. Dawson Memorial Hospital, la Cour suprême de la Nouvelle-Écosse a réduit la longueur du préavis en tenant compte des mauvaises relations qu'entretenait le réclamant avec certains membres du personnel ${ }^{60}$. Cette façon de faire pourrait être suivie au Québec dans la détermination de la durée raisonnable d'un préavis ${ }^{61}$.

La durée du préavis auquel a droit un salarié non cadre est déterminée au Québec par 1'article 82 de la Loi sur les normes du travail ${ }^{62}$. L'article 83 de la même loi précise que le salarié n'aura pas droit au préavis de l'article 82 s'il est congédié pour une faute grave. La jurisprudence a interprété l'expression "faute grave» comme incluant non seulement le manquement tellement grave qu'il justifie une rupture immédiate du contrat mais aussi «une série de faits de moindre importance ou [...] un comportement que 
répétera le salarié malgré un ou des avertissements sérieux» ${ }^{63}$. Nous croyons que ce second volet de la notion de "faute grave» comprend un manquement du salarié à son obligation de civilité. Le congédiement ne sera justifié qu'après l'échec des interventions successives et graduelles de l'employeur.

Le manque de flexibilité du droit commun en matière de mesures disciplinaires et de congédiement confère une importance particulière à la période d'essai dont peuvent convenir les parties. Généralement, cette période permet à l'employeur de vérifier les qualifications et aptitudes du salarié en regard de son emploi et le libère de l'obligation d'établir l'existence d'une cause telle que décrite ci-haut dans l'éventualité d'un congédiement. Ainsi, l'employeur observera de quelle façon le salarié s'intègre à sa nouvelle équipe de travail. $\mathrm{Si}$, à la fin de la période d'essai, l'employeur décèle ou anticipe simplement des problèmes de relations interpersonnelles, il pourra rompre le lien d'emploi. Nous avons vu que les mêmes tensions interpersonnelles ne constitueront pas nécessairement une cause valable de congédiement au-delà de la période d'essai.

Dans Ritchie c. Intercontinental Packers Ltd. ${ }^{64}$, la Court of Queen's Bench de la Saskatchewan confirma le congédiement en cours de période d'essai d'un directeur des ressources humaines. L'employeur avait réagi ainsi à la suite de nombreuses plaintes de l'administration et des membres du personnel à l'endroit de l'arrogance, de l'intransigeance et du défaut du réclamant d'écouter le point de vue des autres. La Cour, en rendant sa décision, énonça le principe suivant:

«... if a probationary employee is fired, the employer must show that he was unsuitable for the job. In doing so, he can justify his conclusion by considering not only job performance but other factors such as character and compatibility. ${ }^{65}$

En d'autres termes, le congédiement d'un salarié à l'essai peut être justifié aussi bien par une faute, par son incompétence ou son manque de qualification que par une appréhension raisonnable qu'il devienne une source de conflit.

Toutefois, seules les règles de discipline développées dans une convention collective fournissent vraiment à l'employeur les moyens de remédier aux tensions entre salariés. Remarquons que ces principes disciplinaires ont été importés en grande partie dans le secteur des rapports individuels de travail par l'intermédiaire des arbitres agissant en vertu de la Loi sur les normes du travail du Québec et de la Partie III du Code canadien du travail $^{66}$. Cette discipline vise essentiellement à corriger le comportement déviant du salarié et, à ce titre, la sanction imposée par l'employeur doit être proportionnelle à la gravité de la faute reprochée au salarié.

Comme le droit commun, la jurisprudence arbitrale ne reconnait pas à l'employeur le pouvoir de congédier sur-le-champ un salarié pour avoir manqué à son obligation de civilité. Il devra plutôt recourir à des sanctions de moindre importance pour signifier au salarié la nécessité d'améliorer son attitude à l'endroit des autres membres du personnel. Ce n'est que dans le cas où il n'y a aucune amélioration après plusieurs mises en garde qu'un congédiement sera justifié. Ceci explique le fait que beaucoup de congédiements imposés pour manque de civilité et contestés devant des arbitres sont cassés et remplacés par des mesures disciplinaires de moindre importance ${ }^{67}$. 
La réparation d'un congédiement imposé à la suite d'un conflit interpersonnel entre salariés et déclaré injuste par l'arbitre pose un problème particulier. L'instruction du dossier et la décision de l'arbitre ne parviennent généralement pas à dissiper le malaise existant, loin de là. Dès lors, la réintégration, remède généralement imposé à la suite d'un congédiement injuste, s'avère particulièrement inadéquate. C'est pourquoi plusieurs arbitres préfèrent ordonner une simple indemnité compensatoire sans réintégration ${ }^{68}$.

L'employeur ne pourra intervenir disciplinairement que s'il y a manquement du salarié à son obligation de civilité. Or, pour qu'un tel manquement survienne, il faut qu'il y ait comportement fautif du salarié. On peut cependant penser à des situations où le salarié est rejeté de son groupe de travail alors même qu'il n'y a aucune faute ou mauvaise volonté de sa part. Ainsi, dans l'affaire Childs c. The Royal Bank of Canada ${ }^{69}$, les absences répétées pour cause de maladie de la réclamante avaient provoqué un profond ressentiment à son endroit de la part de ses compagnes de travail, celles-ci devant assumer une partie de son travail en son absence. La détérioration du climat de travail amena l'employeur à congédier la réclamante, une excellente employée par ailleurs. L'arbitre annula le congédiement et accorda une indemnité compensatrice sans réintégration. Mais comment donc l'employeur aurait-il pu réagir pour demeurer à l'intérieur des limites permises par le droit?

En vertu du droit commun, nous l'avons vu, l'employeur pourra rompre le lien d'emploi moyennant un préavis (dont la longueur doit respecter les normes légales) en autant que le contrat de travail soit à durée indéterminée. Dans le cas contraire, l'employeur devra attendre l'arrivée du terme. Le cas se complique lorsque le salarié est admissible au recours. de l'article 61.5 du Code canadien du travail, Partie III, (après un an de service continu) ou à celui des articles 124 et seq. de la Loi sur les normes du travail du Québec (après cinq ans de service continu). Ces dispositions exigent que l'employeur ait une cause juste et suffisante pour congédier un salarié. Donner un préavis ne le libère pas de son obligation de démontrer cette cause ${ }^{70}$. L'employeur se trouve donc dans une impasse.

C'est une situation semblable qui amena l'arbitre Borowicz souligner ceci dans l'affaire Childs:

«At the time when the decision to dismiss the complainant was made, it may have been the only alternative available to the Bank. In that event, with reasonable notice, or with salary in lieu therof, it was open to the Bank to terminate her contract»..1

Il demeure encore difficile de déterminer si une telle opinion sera partagée ou non par la jurisprudence. En pratique cependant, les arbitres acquiescent implicitement à une telle solution en octroyant une indemnité sans ordonner la réintégration du salarié injustement congédié.

Dans le secteur régi par convention collective, le même problème survient. En effet, l'employeur renonce généralement à son pouvoir de résiliation unilatérale avec préavis. C'est pourquoi, dans certains cas de conflits interpersonnels, l'employeur pourra tenter de déplacer le salarié, ce qui peut d'ailleurs être accepté par l'arbitre ${ }^{72}$. Si la relocalisation est impossible, 
l'employeur devrait tenter de régler le problème tout en maintenant le salarié à l'emploi. Il nous semble en effet difficile de permettre à l'employeur de se départir d'un salarié, même avec préavis, alors qu'il n'y a pas de cause juste et suffisante de congédiement. La question demeure toutefois ouverte.

\section{CONCLUSION}

Cette étude fait ressortir le fait que, le salarié étant parfois en situation d'interrelation avec d'autres, au moment de fournir sa prestation de travail, il en résulte des règles particulières que nous avons traduites en une obligation de civilité. À celles-ci correspondent, à l'occasion, des manquements auxquels peut réagir l'employeur. Cependant, l'étude de la jurisprudence dans son état inachevé - révèle un cadre juridique différent de celui auquel nous sommes habitués, celui-ci ne prenant en compte que les actes individuels d'un salarié pris isolément (sauf dans le domaine des manquements collectifs dont le cadre juridique est également en voie d'élaboration).

L'obligation de civilité, comme nous l'avons définie, pose un problème particulier en ce qu'elle présuppose une participation de tiers-salariés dans la conception même du manquement reproché.

Ainsi, la réaction des compagnons de travail au comportement déviant devient un élément constitutif de la faute présumée. L'effet qui résulte de cette interaction sur le climat dans le groupe social en est l'élément déterminant. En l'absence de cet élément, l'acte reproché retombe dans la catégorie des fautes individuelles, qui comprend même celles qui se font au détriment de tiers-salariés, par exemple le vol de biens appartenant à des compagnons de travail ou l'assaut.

Il est vrai que ces fautes peuvent provoquer aussi l'ostracisme de la part du groupe. L'on voit par là que la notion de groupe social et le rôle qu'y joue l'individu sont le fondement même de la notion d'obligation de civilité.

Par-delà les problèmes strictement juridiques qui se rattachent à cette obligation, on en discerne d'extra-juridiques qui nécessiteraient l'éclairage d'autres disciplines.

Ainsi, dans un groupe syndiqué (hypothèse que nous avons écartée) on peut se demander comment devrait ou pourrait réagir le syndicat, divisé contre lui-même en pareille matière. Comment concilier l'obligation de représenter au mieux les intérêts du groupe et l'obligation que lui fait le Code du travail de représenter justement l'individu frappé de sanction disciplinaire?

Par ailleurs, comment l'employeur devrait-il réagir, lui qui doit traiter équitablement chaque salarié, donc qui ne peut sacrifier l'individu en butte aux manifestations de reiet du groupe? Heureux le chef d'entreprise aui. face à pareille difficulté, peut recourir à l'échappatoire de la mutation,

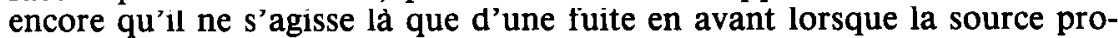
fonde du problème se trouve dans la personnalité même du salarié. Force est de conclure qu'en dernier ressort, ce salarié doit partir s'il est incapable de 
s'amender. Si par contre la discrimination est à l'origine du problème et que le transfert du salarié n'améliore pas la situation, les nuages s'amoncelleront à l'horizon c'est-à-dire que le groupe devra céder, de gré ou de force, sous la contrainte de la législation contre la discrimination, avec la coopération de l'employeur, quel qu'en soit le prix.

\section{NOTE S}

1 Nous postulons, pour les fins de la discussion, qu'il n'y a pas de convention collective ou encore que les obligations fondamentales nées du contrat survivent à l'avènement de la convention collective.

Bien entendu, ce postulat ne résout pas la question de la délicate relation qui existe entre ces deux actes juridiques. Il n'a d'utilité que pour délimiter l'objet de notre étude.

2 Notre étude portera sur les relations entre les salariés exécutants, de même qu'entre ces derniers et les détenteurs de l'autorité hiérarchique dans l'entreprise.

Certes, le cadre a une obligation de solidarité avec ses collègues, mais elle ne fait pas l'objet de notre étude.

3 Dans un contexte de division du travail où, à tout le moins de travail en groupe, on ne peut dissocier le rendement d'un individu de celui des autres. En d'autres mots, le salarié ne remplit pas son obligation de fournir une prestation convenable si, par son comportement perturbateur, il nuit au travail des autres.

4 Le contrat de travail comporte trois éléments essentiels, selon la théorie classique:

i) la subordination juridique du salarié qui lui impose un devoir d'obéissance, quant aux moyens d'exécuier le travail, à l'égard de l'employeur oü de ses représentantants;

ii) la prestation de travail à laquelle se rattache l'obligation de fournir le travail convenu, obligation de diligence en vertu de laquelle le travailleur est tenu de se comporter en bon père de famille, dans l'exécution de ses fonctions;

iii) le paiement du salaire (dont nous n'avons pas parlé jusqu'ici).

5 La civilité, comme nous l'entendons, ne doit pas être confondue avec les règles de comportement identifiant les classes sociales et contribuant à maintenir le fossé qui les sépare. $C f$. Lewis A. COSER, "The Notion of Civility in Contemporary Society», (1980) XXI Archives européennes de sociologie, pp. 3-13.

Une seconde précision s'impose. Dans le cas particulier où le travailleur est régulièrement en rapport avec des tiers - clients, bénéficiaires, contribuables, etc. - on pourrait à juste titre parler aussi d'une obligation de civilité à leur endroit. Ici, nous nous intéresserons aux seules relations interpersonnelles à l'intérieur du groupe de travail.

Dans l'hypothèse où le travailleur est en rapport avec des tiers, son obligation de fournir une prestation convenable se subdivise en deux parties. C'est une obligation conjonctive, à double objet et l'inexécution ou la mauvaise exécution d'une prestation équivaut à l'inexécution de l'ensemble de l'obligation. À l'inverse, la bonne exécution d'une prestation ne compense pas l'exécution déficiente de l'autre. Comme le notait la Cour d'appel de Paris:

«... On ne saurait [...] tenir compte des attestations que monsieur DUMONT fournit en sa faveur. Emanant de divers commerçants, elles ne font état que des bonnes relations que l'intéressé entretenait avec la clientèle, mais sont étrangères à ses difficultés personnelles avec son employeur..."

S.A. des Caves et des Producteurs réunis de Roquefort c. Dumont, Cour d'appel de Paris, le 19 mai 1982, p. 5. 
Sur l'obligation conjonctive, voir Jean-Louis BAUDOUIN, Les obligations, deuxième édition, Y. BLAIS, Cowansville, 1983, $\mathrm{n}^{\circ}$ 804; Alex WEIL et François TERRÉ, Les obligations, deuxième édition, Précis Dalloz, Paris, 1975, $\mathrm{n}^{\circ}$ 924; Gabriel MARTY et Pierre RAYNAUD, Droit civil, tome II, premier volume (Les obligations), Sirey, Paris, 1962, $\mathrm{n}^{\circ} 764$; Marcel PLANIOL et Georges RIPERT, Traité pratique de droit civil français, tome VII (avec le concours de P. Esmein, J. Radouant et G. Gabolde), L.G.D.J., Paris, 1954, nº 1047.

Il est à noter que l'employeur est créancier de l'obligation de civilité envers les tiers et non ces derniers. Elle naît du contrat de travail; il s'agit donc ici ni de stipulation pour autrui, ni de promesse de porte-fort.

Si un tiers en rapports d'affaires avec un employeur a des motifs de se plaindre du comportement d'un salarié, il n'aura qu'à dénoncer le fait au patron qui prendra les mesures qui s'imposent pourvu toutefois que la faute présumée constitue une entorse aux obligations nées du contrat individuel de travail.

Le tiers ne pourra agir de son propre chef que si la conduite du salarié est génératrice de droit e.g. s'il y a faute délictuelle ou infraction criminelle.

6 Il s'agirait en somme de la bonne foi pouvant servir de fondement à un «devoir d'assistance, de collaboration, de coopération, d'aide mutuelle et, à la limite d'amitié et de fraternité»: Gérard Cornu, cité par Jacques GUESTIN, traité de droit civil, Tome II, L.G.D.J., Paris, 1980, p. 142.

L'origine de cette idée remonte à René DEMOGUE, Traité des obligations en général, Tome VI, Librairie Arthur Rousseau, Paris, 1931, pp. 8-10.

7 Nous avons déjà signalé le cas où le groupe rejette l'individu pour des motifs prohibés par la loi e.g. en raison de sa race, de sa religion, etc. Dans de tels cas, à la limite, le groupe doit s'adapter, de gré ou de force, à l'individu. Pour un cas où les tribunaux ont refusé d'entériner le renvoi d'un individu - cadre en l'espèce - rejeté par le groupe en raison de sa langue, voir: Terminal Construction Co. Ltd c. Piscitelli, (1960) B.R. 593. Cette décision est fondée sur le droit commun et non sur la législation protectrice des droits de la personne.

Nous n'aborderons pas non plus le rejet de groupes minoritaires par la majorité, qui déborde nettement le cadre du droit des rapports individuels; voir, par exemple, le cas de la mise au ban de non syndiqués in Perreault c. Gauthier (1897) 28 R.C.S. 241.

8 Cette obligation est mutuelle, mais il n'entre pas dans notre propos de discuter cet aspect de la question.

9 Voir au sujet du champ d'étude de la psychologie sociale: Fred LUTHANS, Organizational Behavior, Third edition, McGraw-Hill Book Company, New York, 1981, pp. 50-51.

10 Voir chez les auteurs suivants la définition du groupe: A.L. ATHOS et R.E. COFFEY, Behavior in Organization: A Multidimensional View, Prentice-Hall Inc., New Jersey, 1968, p. 83; D.R. FORSYTH, An Introduction to Group Dynamics, Brooks/Cole Publishing Company, Monterey, California, 1983, pp. 8-9; J.L. GIBSON, J.M. IVANCEVICH et J.H. DONNELLY Jr., Organization, Structure, Processes, Behavior, Business Publications Inc.; Dallas, Texas, 1973, p. 253; W.C. HAMMER et D.W. ORGAN, Organizational Behavior. An Applied Psychological Approach, Business Publications Inc., Dallas, Texas, 1978, p. 303.

11 Voir à ce sujet: A.L. ATHOS et R.E. COFFEY, op. cit., p. 87; J.L. GIBSON, J.M. IVANCEVICH et J.H. DONNELLY Jr, op. cit., pp. 256-259; F. LUTHANS, op. cit., p. 331; W.C. HAMMER et D.W. ORGAN, op. cit., p. 303.

12 E.H. SCHEIN, Organizational Psychology, Third edition, Prentice-Hall Inc., Englewoods Cliffs, New Jersey, 1980, p. 146.

13 Pour plus de détails sur les facteurs expliquant la formation des groupes informels voir: J.L. GIBSON, J.M. IVANCEVICH et J.H. DONNELLY Jr, op. cit., pp. 256-259.

Par ailleurs, d'un point de vue macro-sociologique, l'avènement de la société postindustrielle et les changements technologiques qui s'y rattachent ont eu un effet indéniable sur 
la formation et le développement des groupes informels au travail, notamment en créant de plus en plus de périodes au cours d'un quart de travail pendant lesquelles les individus ne sont pas directement astreints aux tâches inhérentes à leur poste.

14 Voir au sujet des étapes de la formation d'un groupe: W.C. HAMMER et D.W. ORGAN, op. cit., pp. 304-305; J.L. GIBSON, J.M. IVANCEVICH et J.H. DONNELLY Jr, op. cit., pp. 259-260.

15 Voir pour plus de détails sur les caractéristiques des groupes: W.C. HAMMER et D.W. ORGAN, op. cit., pp. 305-315; J.L. GIBSON, J.M. IVANCEVICH et J.H. DONNELLY Jr, op. cit, pp. 262-263; A.G. ATHOS et R.E. COFFEY, op. cit. , pp. 209-210.

16 Voir chez les auteurs suivants la définition des normes: W.C. HAMMER et D.W. ORGAN, op. cit., p. 313; J.L. GIBSON, J.M. IVANCEVICH et J.H. DONNELLY Jr, op. cit., p. 263; A.L. ATHOS et R.E. COFFEY, op. cit., pp. 97-98.

17 J.R. HACKMAN, «Group Influences on Individuals», in Handbook of Industrial and Organizational Psychology, M.D. DUNNETTE, Editor, Rand McNally College Publishing Company, Chicago, 1976, pp. 1495-1496.

18 Ainsi, il y a des normes auxquelles doit obligatoirement se conformer chaque membre afin de demeurer au sein du groupe (pivotal norms) et d'autres auxquelles le membre doit préférablement se conformer, mais sans toutefois qu'une dérogation de sa part entraîne son rejet du groupe (peripheral norms). Voir E.N. SCHEIN, op. cit., pp. 99-100.

19 Certaines lois fixent également des normes en deçà desquelles l'employeur ne peut aller. Voir par exemple la Loi sur les normes du travail, L.R.Q., c. N-1.1.

20 Par exemple, la norme minimale de rendement fixée par l'employeur trouve parfois son pendant dans la norme maximale fixée par le groupe informel. Alors que l'employeur incite chaque salarié à dépasser la norme, le groupe s'assure qu'aucun membre ne s'en écarte sensiblement.

21 Le lecteur trouvera en seconde partie des exemples tirés de la jurisprudence.

22 F. LUTHANS, op. cit., p. 127.

23 E.H. SCHEIN, op. cit., pp. 20-21.

24 Ibid., p. 22.

25 S.R.C., 1970, c. L-1.

26 L.R.Q., c. N-1.1.

27 La jurisprudence canadienne est ici pertinente puisque l'employment contract de common law est, à peu de choses près, similaires à notre contrat individuel de travail de droit civil. Sur l'employment contract, voir de façon générale: I. CHRISTIE, Employment Law in Canada, Butterworths, Toronto, 1980, 518 pages.

28 Voir à titre d'exemples, les décisions suivantes: Hôpital St-Jean-de-Dieu c. MarieClaire Charbonneau, A.H.P.Q. 325-03, le 6 février 1969, (C. Beaulieu, président); Hôpital StJean-de-Dieu c. Syndicat national des employés de l'Hopital St-Jean-de-Dieu et al., (1970) S.A.G. 1, (V. Melançon, arbitre); La Société de publication du Journal de Montréal c. Le Syndicat des travailleurs de l'information du Journal de Montréal, (1976), S.A.G. 1149, (J.P. Lalancette, arbitre); Centre hospitalier Ste-Jeanne d'Arc c. Syndicat des employés d'hôpitaux de Montréal, D.T.E. 83 T-132, le 23 décembre 1982, (J.-P. Lussier, arbitre); Donaghue c. South West Air Ltd., décision non publiée rendue selon l'article 61.5 du Code canadien du travail, le 7 mai 1980, (G. Brent, arbitre); Johnson c. P. Dickson Trucking Ltd., décision non publiée rendue selon l'article 61.5 du Code canadien du travail, le 15 novembre 1983, (F.S. Borowicz, arbitre); M. Manolopoulos c. B.P. Pétroles Ltée, SA-124-83-182, (décision non publiée rendue selon l'article 124 de la Loi sur les normes du travail), le 9 septembre 1983, (G. Jutras, arbitre).

29 Fonceca c. Mc Donnell Douglas Can. Ltd., Canadian Cases on Employment Law 51, le 5 avril 1983. (Ce recueil est ci-après désigné par le sigle C.C.E.L.)

30 Ibid. 
31 Voir en ce sens: Hôpital St-Jean-de-Dieu c. Marie-Claire Charbonneau, précitée à la note 28; Hopital St-Luc: grief de Gaston Boilard, A.H.P.Q. 325-03-7, le 4 novembre 1970, (D. Gagnon, arbitre); Hôpital du Sacré-Coeur de Montréal, grief d'Hubert Tourigny, A.H.P.Q. 327-06-07, le 21 mai 1980, (J.-J. Turcotte, président); Pépinière Bourbeau Inc. c. Normand Boies (décision non publiée rendue en vertu de l'article 124 de la Loi sur les normes du travail), SA-124-83-186, le 15 septembre 1983, (M. Langlois, arbitre).

32 La Société de publication du Journal de Montréal, précitée à la note 28; Association Indépendante des Employé(es) de Thursday et Hôtel de la Montagne c. Thursday's Restaurant and Bar Inc., non publiée, le 12 septembre 1985, D.T.E. 851-805, (C.H. Foisy, arbitre); Johnson c. P. Dickson Trucking Ltd., précitée à la note 28.

33 (1975) 9 L.A.C. (2d) 326, (R.D. Abbott, prés.) à la page 331. Voir aussi: Manolopoulos c. B.P. Pétroles Ltée, précitée à la note 28.

34 Centre hospitalier Ste-Jeanne D'Arc, précitée à la note 28

35 Hôpital St-Jean-de-Dieu c. Syndicat national des employés de l'Hôpital St-Jean-deDieu, précitée à la note 28 .

36 Micheline Guenette c. Eng. Po Inc., SA-124-83-81, décision non publiée, rendue en vertu de l'article 124 de la Loi sur les normes du travail, le 20 avril 1983, (M. Bergevin, arbitre).

37 Dans l'affaire Banville c. P.G. Du Québec (C. Supérieure, Hauterive, le 9 janvier 1984, D.T.E. 84T-172), M. le juge Trotier mentionne ceci:

«... l'on sait que l'inhabilité pour un employé d'être en relation harmonieuse avec son patron ne constitue pas en soi une cause de congédiement.» (À la page 4 du jugement). Dans le même sens: Bibeau c. Centre local des services communautaires du Marigot, C.S. (Montréal), le 27 novembre 1985, D.T.E. 86T-2.

Voir également les décisions suivantes rendues selon les articles 124 et seq. de la Loi sur les normes du travail du Québec ou 61.5 de la Partie III du Code canadien du travail: $T$. Beaudoin c. Visionic Inc., SA-124-81-37, le 29 juillet 1981, (J.G. Michaud, arbitre); J. Campeau c. Torpedo Ltée., SA-124-82-33, le 24 mars 1982, (R. Marcheterre, arbitre); $A$. Tanguay c. J.-P. Jetté, SA-124-82-84, le 16 juin 1982, (M. Bolduc, arbitre)' T. Chouinard c. Coopérants (Société mutuelle d'assurance-vie), SA-124-83-09, le 6 janvier 1983 (M. Bolduc, arbitre); $R$. Westbury c. Kingsway Transport Ltd., le 20 décembre 1979, (E.E. Palmer, arbitre); S.J. Brown c. Futura Airlines Limited, le 6 mai 1982 (B.M. Greyell, arbitre).

38 Houle c. La Fédération de l'U.P.A. de Sherbrooke et al., (décision non publiée rendue selon l'article 124 de la Loi sur les normes du travail), SA-124-84-40, le 8 février 1984, (R. Marcheterre, arbitre).

39 Thorneloe c. La Commission scolaire régionale Eastern Townships, C.S. (StFrançois), le 23 octobre 1983, D.T.E. 84T-870.

40 Hôpital St-Jean c. L'Association des employés de l'Hôpital de St-Jean Inc., (1963) R.D.T. 97, (R. Ouimet, président); $R$. Huneault c. Central Mortgage and Housing Corporation, (décision non publiée rendue en vertu de l'article 61.5, Partie III, Code canadien $d u$ travail, le 27 août 1979, (M. Cohen, arbitre).

41 Armstrong c. Atlantic Trades Limited et al, 46 N.S.R. (2d) 117 (Nova Scotia Supreme Court, le 5 juin 1981); Speedy Heavy Hauling Ltd c. E. Wilson, (décision non publiée rendue en vertu de l'article 61.5, Partie III, Code canadien du travail, le 12 septembre 1983, (G.M. Morrison, arbitre); Pépinière Bourbeau Inc. c. Normand Boris, précitée à la note 31.

Dans Maheu c. Catalytic Enterprises Ltd., (Cour supérieure, Montréal, le 8 juin 1984, D.T.E. 84T-636), le tribunal déclara injustifié un congédiement imposé à un surintendant parce que les trois contremaîtres refusaient dorénavant de travailler sous ses ordres. La Cour mentionna expressément que le demandeur n'avait rien fait de répréhensible et que le conflit de personnalités qui l'opposait à ses contremaîtres n'avait pas sa source dans les rapports du travail.

42 Code du travail, L.R.Q., c. C-27, art. 100.2 
43 L'Association des travailleurs en fourrure de Montréal c. D.H. Grosvenor Inc., non publiée, le 12 juin 1980, (A. Madras, arbitre); Charron c. Madras, (1981) R.L. 304 (C.S.); Charron c. Madras et al, Cour d'appel, numéro 500-09-000271-817, le 7 novembre 1983. Dans son opinion, Monsieur le juge Jacques apporte la réserve suivante:

«... il ne faudrait pas que cette décision soit considérée comme un précédent qui aurait pour effet d'obliger une partie à faire une preuve disculpatoire sur simple dépôt par la partie adverse d'un document racontant un fait quelconque.» (à la page 2).

$44 E$. Bisonnette c. Bélanger et Garceau, (décision non publiée rendue en vertu de l'article 124 de la Loi sur les normes du travail, SA-124-84-122, le 19 mai 1984 (voir à la page 13 de la décision).

45 Hôpital du Sacré-Coeur de Montréal, précitée à la note 31, à la page 28. Voir aussi: Association Indépendante des Employés de Thursday et Hôtel de la Montagne, précitée à la note 32 (dans laquelle l'arbitre substitue une suspension de deux semaines au congédiement). Dans Marché Montour Inc. c. G. Fournier, (décision non publiée rendue selon l'article 124 de la Loi sur les normes du travail), SA-124-83-213, le 31 octobre 1983, l'arbitre R. Marcheterre écarte une pétition signée par les salariés comme preuve de la mauvaise conduite du réclamant. Cette pétition avait été signée près de six mois après le renvoi du salarié et mentionnait que, dans l'éventualité de son retour au travail, certains salariés quitteraient leur emploi. L'arbitre ajoute cependant que la pétition l'avait incité à ne pas ordonner la réintégration.

$46 R E$ Continuous Colour Coat Ltd., précitée à note 33; Association Indépendante de Thursday et Hôtel de la Montagne, précitée à la note 32.

47 Voir: M. DESPAX, «La vie extra-professionnelle du salarié et son incidence sur le contrat de travail», J.C.P., 1963, II, 1776.

48 Hôpital St-Luc, grief de G. Boilard, précitée à la note 31. Voir aussi les propos de la Cour suprême du Canada dans Corporation de l'Hôpital Bellechasse c. L. Pilotte et al, (1975) 2 R.C.S. 454 (particulièrement à la page 463).

49 Voir: Régie Nationale Renault c. Fontaine, Cour de Cassation (France), le 2 février 1950, publiée dans (1950) Droit social, 171.

so Houle c. La Fédération de l'U.P.A. de Sherbrooke et al, précitée à la note 38.

51 Voir supra le texte accompagnant les notes 30 iे 41.

52 Ainsi, l'article 10 de la Charte des droits et libertés de la personne du Québec (L.R.Q., c. C-12) identifie les critères suivants sur lesquels aucune discrimination ne peut être fondée: la race, la couleur, le sexe, la grossesse, l'orientation sexuelle, l'état civil, l'âge (sauf dans la mesure prévue par la loi), la religion, les convictions politiques, la langue, l'origine ethnique ou nationale, la condition sociale, le handicap ou l'utilisation d'un moyen pour pallier à ce handicap. Voir aussi l'article 3(1) de la Loi canadienne sur les droits de la personne, S.C. (1976-77), c.33 (telle qu'amendée), pour les entreprises de juridiction fédérale.

53 Voir par exemple Shaffer c. Treasury Board of Canada, 84 CLLC 17 019, décision rendue en appel par un tribunal des droits de la personne en vertu de la Loi canadienne sur les droits de la personne, citée à la note précédente.

54 Voir l'article 10.1 de la Charte des droits et libertés de la personne du Québec et l'article 13.1 (1) et (2) de la Loi canadienne sur les droits de la personne, précitées à la note 52.

Voir aussi la décision de la Cour supérieure dans G. Foisy c. Bell Canada, (1984) C.S. 1164 , où Madame le juge $L$. Mailhot accueille une action en dommages-intérêts instituée contre l'employeur par une salariée à la suite de harcèlement sexuel par son supérieur immédiat. On y lit notamment que:

"Le tribunal est d'avis que cette poursuite contre l'employeur est recevable, Bell étant responsable des gestes fautifs posés par ses préposés, administrateurs et cadres, quelle que soit leur position hiérarchique, (à la page 1172).»

Voir cependant à l'effet contraire: Brennan and Her Majesty The Queen et al., Cour d'appel fédérale, le 18 février 1985, M. le juge en chef et M.M. les juges Pratte et MacGuigan, en particulier à la p. 21 . 
On y affirme aussi que l'employeur n'a aucune obligation de prendre des mesures en vue d'empêcher le harcèlement sexuel; par ailleurs, des lettres et pétitions ayant circulé après que la salariée eût déposé sa plainte n'avaient - semble-t-il - aucune valeur (en réalité, la Cour approuve l'employeur d'avoir déchiré ces documents en présence de la salariée et d'un représentant syndical). Enfin le déplacement de la réclamante dans un autre département fut jugé non pertinent parce que postérieur aux faits ayant donné naissance à la dénonciation.

Pour une étude de l'étendue du phénomène du harcèlement sexuel au travail, voir $\mathrm{D}$. SAVOIE, Le harcèlement sexuel au travail et les femmes québécoises, mémoire de maîtrise, Ecole de relations industrielles, Université de Montréal, novembre 1984, 524 pages.

55 Code canadien du travail, Partie III, S.R.C. 1970, c. L-1, amendé par la Loi modifiant le Code canadien du travail et la Loi sur l'administration financière, S.C. 1983-84, c. 39, article 12.

56 Tel que mentionné à la note 1 , nous postulons que les obligations fondamentales nées du contrat survivent à l'avènement de la convention collective. Aucune des décisions arbitrales consultées ne traite de la source de l'obligation de civilité du salarié.

57 Voir, parmi d'autres, sur ce point: R. BLOUIN, «Notion de cause juste et suffisante en contexte de congédiement», (1981) 41 Revue du Barreau, 807, aux pp. 808 à 813 .

58 Précitée à la note 39. Voir aussi: Banville c. P.G. du Québec, précitée à la note 37; Maheu c. Catalytic Enterprises Limited, précitée à la note 41, D. HARRIS, Wrongful Dismissal, Fourth edition, Richard De Boo, Don Mills (Ontario), 1984, aux pages 3-137, et 3-138.

59 HARRIS, ibid; DESPAX, loc. cit., supra note 47, paragraphe $n^{\circ} 41$; S.T. ANDERMAN, The Law of Unfair Dismissal, Butterworths, London, 1978, 378 pages, à la page 165.

60 (1978) 3 A.C.W.S. 222, citée dans HARRIS, op. cit., supra note 58, à la page 3-147. Voir aussi la présentation de cette tendance que fait HARRIS aux pages 3-146 a 3-153 de son ouvrage. Cependant, la notion de «near cause» est en voie d'être écartée par la plus récente jurisprudence: $c f$. (1986) 2 Wrongul dismissal, $\mathrm{n}^{\circ} 1$, A Monthly Supplement to Lancaster Labor Law Service, pp. 1-3.

61 Voir: G. AUDET et R. BONHOMME, Le congédiement en droit québécois, Les Éditions Yvon Blais Inc., Cowansville, 1985, 236 pages, aux pp. 35 et 36; Malabre c. Idi Electric (Canada) Ltd, (1984) C.S. 563, commentée par C. D'AOUST, «Le pouvoir réparateur du juge de droit commun en matière de congédiement», (1984) 15 R.D.U.S. 233.

62 L.R.Q., c. N-1.1

63 Commission des normes du travail c. Beverini Inc., Cour provinciale (Montréal), le 16 juillet 1982, J.E. 82-967, à la page 23 du jugement. Voir aussi AUDET et BONHOMME, op. cit., supra note 61, aux pages 169 et 170 .

642 C.C.E.L. 147, le 22 janvier 1982.

65 Ibid., à la page 152. La Cour suprême du Canada, dans Jacmain c. Le Procureur général du Canada et al., (1978) R.C.S. 15, mentionnait que:

«Le mauvais comportement de l'employé, son attitude acerbe, son ajustement défectueux à son entourage constituent pour son chef des raisons valables de ne pas vouloir lui accorder un emploi permanent dans son service.» (Á la page 38)

Voir aussi, en jurisprudence arbitrale, Nordair Inc. c. International Association of Machinists and Aerospace Workers, Lodge number 2309, non publiée, le 28 octobre 1985, (H. Frumkin, arbitre).

66 Voir P. LAPORTE, Le recours d̀ l'encontre des congédiements sans cause juste et suffisante, Wilson et Lafleur Ltée, Montréal, 1985, 211 pages, aux pp. 139 et seq.; G. TRUDEAU, Statutory Protection Against Unjust Dismissal for Unorganized Workers, thèse de doctorat, Harvard Law School, 1985, 476 pages, aux pp. 136 et seq.

Pour une étude du droit disciplinaire sous l'empire d'une convention collective, voir: C. D'AOUST, L. LECLERC et G. TRUDEAU, Les mesures disciplinaires: étude jurisprudentielle et doctrinale, monographie $\mathrm{n}^{\circ} 13$, Ecole de relations industrielles, Université de Montréal, 1982, 484 pages. 
67 À titre d'exemples, voir: Hôpital de Montréal pour enfants c. Infirmiers et Infirmières Unis Inc., (non publiée), le 19 septembre 1983, (F.G. Fortier, arbitre); Association Indépendante des Employes(es) de Thursday et Hôtel de la Montagne c. Thursday's Restaurant and Bar Inc., précitée à la note 32. Les décisions suivantes ont été rendues en vertu des articles 124 et seq. de la Loi sur les normes du travail: Delta Net Inc. c. P. Croteau, SA-124-81-77, le 20 novembre 1981, (J. Dupont, arbitre); Meubles Roxton Ltée c. J.-P. Vaillancourt, SA-124-81-19, le 23 février 1982, (J.-P. Lemieux, arbitre); C. Alain-Mariage c. Savard, Cayer et Ass., SA-124-82-61, le 19 mai 1982, (C. Rondeau, arbitre); Marché Montour Inc. c. $O$. Fournier, précitée à la note 45. Les décisions suivantes ont été rendues en vertu de l'article 61.5 de la Partie III du Code canadien du travail: L. Thompson c. Innotech Aviation Ltd., le 3 décembre 1979, (M. Thompson, arbitre); A. Roberts c. The Bank of Nova Scotia, le 6 décembre 1979, (G.W. Adams, arbitre); G. Clark c. Weatherford Oil Tool Co. Ltd., le 19 mars 1982, (W.P. Moore, arbitre); W. Jordan c. Paul Band Administration, le 9 avril 1985, (C.B. Williams, arbitre).

68 Syndicat de l'Institut canadien d'éducation des adultes (C.S.N.) c. Institut canadien d'éducation des adultes, (non publiée), le 19 mai 1984, (P.Mackay, arbitre). L'indemnité sans réintégration se rencontre beaucoup plus fréquemment dans les décisions arbitrales s'appliquant dans un contexte non syndiqué. Dans plusieurs cas, le salarié lui-même renonce à la réintégration. Les décisions suivantes ont été rendues en vertu de l'article 124 et seq de la $L o i$ sur les normes du travail du Québec: $R$. Campbell c. Maislin Realties, a division of Maislin Transport Ltd., SA-124-83-05, le 5 janvier 1983, (M. Bergevin, arbitre); G. Boucher c. Brasserie Pietro, SA-124-83-43, le 24 février 1983, (M. Bolduc, arbitre); A. Corbeil c. Simpsons-Sears Ltée, SA-124-83-46, le 26 février 1983, (J.-P. Lalancette, arbitre); Pépinière Bourbeau Inc. c. N. Boris, précitée à la note 31; Marché Montour Inc. c. G. Fournier, précitée à la note 45. Les décisions suivantes ont été rendues en vertu de l'article 61.5 de la Partie III du Code canadien du travail: Canada Products c. David Griffiths, le 24 octobre 1983, (J.B. Rose, arbitre); Canadian National Railways c. L. Taylor, le 28 mars 1984, (C.P. Clarke, arbitre), $R$. Davidson c. Radio IWC Ltd., le 8 novembre 1984, (E.B. Jolliffe, arbitre); W. Jordan c. Paul Band Administration, précitée à la note 67.

69 Décision non publiée rendue en vertu de l'article 61.5 de la Partie III du Code canadien du travail, le 27 février 1981, (P.S. Borowicz, arbitre).

$70 M$. Thisdel c. Radio Futura Ltée, (décision non publiée rendue selon l'article $61.5 \mathrm{du}$ Code canadien du travail), le 17 mai 1982 (P. Jasmin arbitre). En ce qui concerne l'article 124 de la Loi sur les normes du travail du Québec, voir: Union des producteurs agricoles c. Martin, (1984), C.S. 724.

71 L.J. Childs c. The Royal Bank of Canada, précitée à la note 69 , à la page 32 du jugement. Même si l'arbitre n'a pas affirmé sa position aussi clairement, la décision suivante pourrait aussi être interprétée comme permettant la rupture du contrat en donnant un préavis suffisant: The Hunter's Horn c. H.P. Moser, (décision rendue en vertu de la Loi sur les normes du travai), SA-124-82-149, le 29 septembre 1982, (P. Imbeau, arbitre).

72 Alliances des professeurs de Montréal c. Commission des Écoles catholiques de Montréal, (1978) S.A.G. 1121, (J.M. Morency, président). Voir aussi, dans cette perspective: Leduc c. UQUAM, (non publiée), le 23 février 1984, (G.E. Dulude, arbitre). Dans ces deux cas les arbitres ont qualifié d'«administratifs» les déplacements imposés aux salariés. 


\section{The Employee's Duty to Get Along}

This essay deals with the employee's so-called «ability to get along» and its correlative «duty to cooperate». The obligation to perform agreed tasks is the fundamental object of the employment contract; however, the authors stress the employee's relations with third parties - fellow workers, management representatives and customers - and the effect his unsatisfactory behaviour may have on his own performance or that of the group he has to work with. (Relationships with customers are ignored for the present purposes; in this respect, attention is drawn to footnote 5).

The common approach - derived from the civil law (and for that matter the common law) concept of master and servant - conceives the duty to perform adequately as one person's obligation towards another. However, such a reasoning conceals the interdependance of individual tasks performed in a social environment, whether or not it stems from the division of labour.

Part One recollects the main features of group and individual behaviour in a social context. Social psychology teaches that such groups develop various norms of conduct and that individual deviation may generate rejection of the employee and even, work havoc in an environment otherwise peaceful and harmonious. Therefore, a newcomer has a duty to accomodate himself to fellow employees and to keep behaving in accordance with the group's expectations. This obligation, we believe, is inherent to the nature of the employment contract as determined by article 1024 of Civil code.

Of course, there are limits to the demands that a group may impose on an individual, the most important being set by the law, especially statutes protecting human rights and prohibiting specific infringements, for instance sexual harassment.

The current state of the law is developed in Part Two, as it evolved in judicial and arbitral decisions and also in statutory provisions.

Emphasis is put on Québec law, with occasional reference to federal and other provincial law. Our analysis, we believe, is readily transposable in both legal systems. 